\title{
Superconductivity and Critical Field Strengths of Palladium-Boron-Hydrogen Alloys
}

\author{
H. Brodowsky and J. Fleischhauer \\ Institut für Physikalische Chemie der Christian-Albrechts-Universität zu Kiel
}

Z. Naturforsch. 38a, 676-679 (1983); received June 4, 1982

Transition temperatures and critical field strengths have been observed on superconducting palladium-boron-hydrogen alloys with different boron to hydrogen ratios. Homogeneous samples, prepared by equilibrium methods, exhibit superconductivity above $3.5 \mathrm{~K}$ for boron contents less than $x=0.07$ ( $x=$ mole boron/mole palladium). Palladium-boron alloys need less hydrogen for reaching a fixed transition temperature than boron free alloys. Critical field strengths for $T_{\mathrm{c}}=0 \mathrm{~K}$ increase linearly with increasing boron content and reach $3.3 \cdot 10^{5} \mathrm{~A} / \mathrm{m}$ at $x=0.044$.

\section{Introduction}

Since the discovery of superconductivity of palladium hydrogen alloys by Skoskiewicz [1] and Stritzker and Buckel [2], many binary and ternary palladium alloys have been examined for superconductivity. It was found that palladium alloys show superconductivity only if at least one light element occupies the octahedral interstitial sites in the palladium fcc lattice.

This behavior has been explained by an extraordinarily large contribution of optical phonons to the electron-phonon coupling constant $[3,4]$. The inverse isotope effect on the transition temperature observed in Pd-H (D) alloys [2] was attributed to the higher force constant for hydrogen atoms compared to deuterium atoms [5]. Particularly high transition temperatures up to $16.6 \mathrm{~K}$ were obtained on the ternary systems $\mathrm{Pd}-\mathrm{Cu}-\mathrm{H}, \mathrm{Pd}-\mathrm{Ag}-\mathrm{H}$, and Pd-Au-H [6].

Only very few experiments have been done so far on superconductivity of palladium alloys with two different elements on interstitial sites. Szafranski et al. [7] found for two different boron concentrations, that palladium samples containing a few percent boron exhibit superconductivity at lower $\mathrm{H}$ concentrations than pure palladium samples.

Based on a number of experiments $[8,9,10]$ and band structure calculations [11] it is well known that hydrogen as well as boron act as electron donors in palladium: hydrogen supplies one, boron three elec-

Reprint requests to Prof. H. Brodowsky, Institut für Physikalische Chemie der Universität Kiel, Olshausenstr. 4060, 2300 Kiel 1, West-Germany. trons to the conduction band of palladium. Upon alloying the band structure is only slightly modified. The main effect is a shift of the Fermi level to higher energies. Thus the palladium-boron-hydrogen system presents the possibility of preparing isoelectronic alloys which differ in boron and in hydrogen concentration. Differences in the transition temperatures of these alloys should show the isolated influence of mass and elastic properties.

In this paper we present the results of some experiments in which the dependence of transition temperatures of palladium-boron-hydrogen alloys on boron to hydrogen ratio has been investigated. Special emphasis was placed on homogenization and accurate determination of $\mathrm{H}$ content, which had not always been possible with the ion implanted samples of earlier work. Critical field strengths of the $\mathrm{Pd}-\mathrm{B}-\mathrm{H}$ system are reported here for the first time.

\section{Experimental}

Palladium foils of $99.99 \%$ purity with an iron content less than $3 \mathrm{ppm}$ (Goodfellow Metals) were used. The palladium foils of approximately $0.125 \times$ $10 \times 20 \mathrm{~mm}^{3}$ were degreased and etched in aqua regia. The samples were charged with boron at $1273 \mathrm{~K}$ by reducing $\mathrm{B}_{2} \mathrm{O}_{3}$ ("suprapur", Merck) in a controlled hydrogen-water vapor atmosphere [12]. The atomic fraction $x=$ mole $\mathrm{B} /$ mole $\mathrm{Pd}$ could be determined with an accuracy of $\Delta x=0.002$ by weighing as well as by X-ray diffraction, using the lattice constant data of Brodowsky and Schaller [13].

The palladium-boron samples were subsequently electrolytically charged with hydrogen. The elec- 
trolyte was nine parts methanol and one part $\mathrm{H}_{2} \mathrm{SO}_{4}$ (conc.) at $220 \mathrm{~K}$. The hydrogen content was measured volumetrically with an accuracy of $\Delta y=0.008$ $(\mathrm{l}=$ mole $\mathrm{H} /$ mole $\mathrm{Pd})$ after thermal decomposition of the samples.

The helium cryostat was of the evaporation type (Oxford Instruments, Type CF 200) with a lowest temperature of $3.5 \mathrm{~K}$. The transition to the superconducting state was measured by a contactless method. An improved version of an ac bridge after Soulen et al. $[14,15]$ allowed sensitive detection of superconductivity and rapid sample changing. The thermocouple at the bottom of the cryostat was calibrated to the temperature inside the measuring coil by the well known transition temperatures of niobium and lead. For measurements of critical field strengths the cryostat was placed inside a magnet.

\section{Results}

$\mathrm{Pd} \mathrm{B}_{x} \mathrm{H}_{y}$ alloys show superconductivity above $3.5 \mathrm{~K}$ at boron contents between $x=0$ and $y=0.07$ (Figure 1). Hydrogen contents between $y=0.959$ and $y=0.640$ were obtained by the electrolytic charging method described above. The highest transition temperatures were found for $x=0.03$.

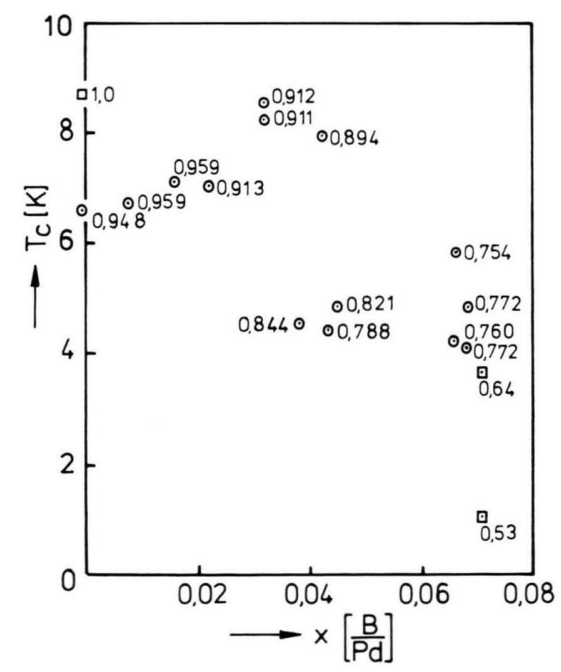

Fig. 1. Transition temperatures of palladium-boron-hydrogen alloys vs. boron content. The hydrogen concentration (mole $\mathrm{H} / \mathrm{mole} \mathrm{Pd}$ ) is indicated for each experimental point. $\square$ : B. Stritzker, J. Becker [6], $\square$ : A. W. Szafranski et al. [7].

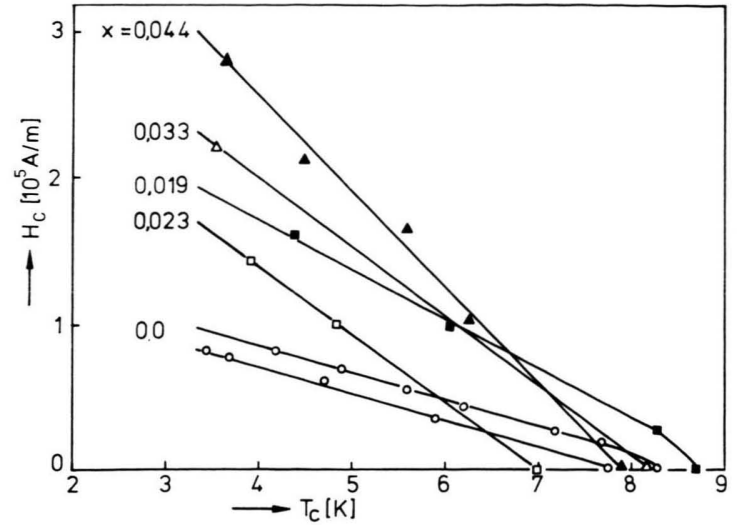

Fig. 2. Critical field strengths vs. transition temperatures of $\mathrm{PdB}_{x} \mathrm{H}_{y}$ alloys.

As had been observed by other authors on Pd-H alloys, the transition curves were rather broad. The width extended from 1.5 to $2.5 \mathrm{~K}$. The temperature at which the transition curve had its maximum slope was taken as the transition temperature. A boron free sample of $\mathrm{PdH}_{0.948}$ had a transition temperature of $6.6 \mathrm{~K}$. This result is in good accordance with results of other authors [16-19].

For samples with low boron content $(x=0$ to $x=0.044)$ the transition temperature was measured as a function of the applied magnetic field (Figure 2). The result of the boron free sample can be compared with the data of Skoskiewicz [17]. He used a linear extrapolation of the $T_{\mathrm{c}}(\mathrm{H})$ curves down to $0 \mathrm{~K}$ and obtained a value of 1800 Oe $\left(1.43 \cdot 10^{5} \mathrm{~A} / \mathrm{m}\right)$, independently of hydrogen content. Using the same method, for purposes of comparison, this result is confirmed here.

\section{Discussion}

\section{Transition temperatures}

There is a definite maximum of transition temperatures for samples near $x=0.03$. However, the samples of the present work differed in hydrogen content as well as in boron content and had different electron concentrations. In order to illustrate the influence of partial replacement of hydrogen by boron, and to eliminate the rather strong influence of a varying electron concentration, the experimental points of Fig. 1 were recalculated for a common valence electron concentration $n_{\mathrm{e}}=1$ by an extrapolation method. 


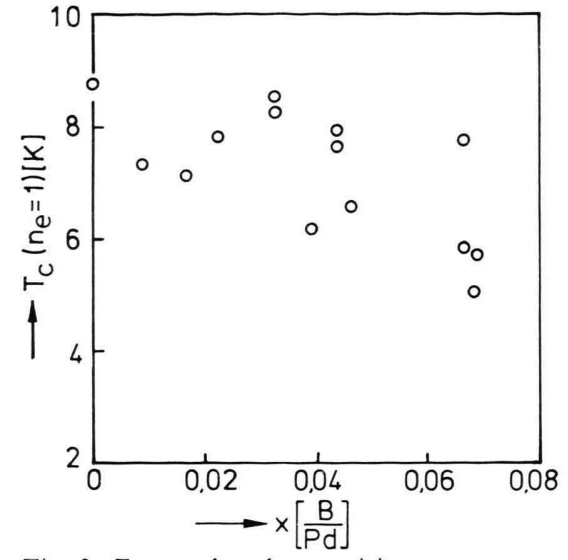

Fig. 3. Extrapolated transition temperatures of $\mathrm{PdB}_{x} \mathrm{H}_{y}$ alloys vs. boron content at valence electron concentration $n_{\mathrm{e}}=3 x+y=1$.

Starting from the actually observed transition temperature, a new $T_{\mathrm{c}}$ was calculated for a slightly increased $\mathrm{H}$ content such that all points in Fig. 3 pertain to the same electron concentration $n_{\mathrm{e}}=1$. Papaconstantopoulos et al. [20] have shown theoretically, that in the Pd-H system the rise of transition temperatures with increasing hydrogen content is almost exclusively a function of the rise of the Fermi level in the $5 \mathrm{~s}$ band region.

The dependence of the transition temperature on electron concentration can be described to a good approximation by a parabolic curve [20]. This behavior is confirmed by experiments for $\mathrm{Pd}-\mathrm{H}$ alloys [17]. The analysis of thermodynamical data [12] and susceptibility measurements [8] shows, that at least in the region of low boron concentration the rise of the Fermi level is identical for $\mathrm{Pd}-\mathrm{H}, \mathrm{Pd}-\mathrm{B}$ and Pd-B-H alloys. Thus the following extrapolation procedure seems to be appropriate: the curve $T_{\mathrm{c}}(y)$ for $\mathrm{PdH}_{\mathrm{r}}$ is shifted vertically, parallel to the $T_{\mathrm{c}}$ axis until an experimental transition temperature lies on that curve (i.e. the constant term in the parabola is fitted to the transition temperature of a $\mathrm{Pd}-\mathrm{B}_{x} \mathrm{H}_{y}$ alloy). On the shifted parabola the new transition temperature can be found corresponding to an electron concentration $n_{\mathrm{e}}=1$. This $T_{\mathrm{c}}$ is expected to be identical to that of a Pd-B alloy, charged with hydrogen up to $n_{\mathrm{e}}=1$. This extrapolation of transition temperatures is justified by the experimental evidence $[8,12]$ that the Fermi energy and the density of states at the Fermi edge do not depend on the hydrogen to boron ratio, but only on the electron concentration. The model of Switendick
[11] shows a strong influence of interatomic distances on the electronic band structure. However, this effect should be very small for isoelectronic Pd-B-H- alloys, because one boron atom with a lattice expansion of $(1 / a)(\partial a / \partial x)=0.174$ [13] replaces three hydrogen atoms with a lattice expansion of $(1 / a)(\partial a / \partial y)=0.059[21]$.

Figure 3 shows the extrapolated transition temperatures. The rise of $T_{\mathrm{c}}$ from $x=0$ to $x=0.03$ has practically disappeared. The large scatter of the data is mainly attributed to the limited accuracy in the determination of hydrogen and boron content.

Apparently the palladium boron alloys need less hydrogen for reaching a fixed transition temperature than boron-free alloys. But the maximum transition temperature (for $n_{\mathrm{e}}=1$ ) decreases with increasing boron content, probably even in the region of low boron concentration $(x<0.03)$. This seems to be predominantly a "normal" dependence of $T_{\mathrm{c}}$ on the masses of atoms, which take part in the electron phonon coupling via optical phonons. Though the force constant, which holds the boron atoms at the octahedral interstitial sites, is about three times higher than the hydrogen force constant [22], no effect analogous to the inverse isotope or mass effect is observed as in $\mathrm{Pd}-\mathrm{H}(\mathrm{D})$ alloys. A pure mass effect is not expected, to begin with, due to the scaling up of the force constant, among other differences between the Pd-B and Pd-H systems.

Preliminary calculations confirm this general trend [15]. More detailed calculations require information on the electron-phonon-interaction in Pd-B-H alloys and on the phonon density of states and are to be presented in connection with the results of ongoing neutron experiments on Pd-B lattice dynamics.

\section{Critical field strengths}

On the basis of the experimental points of the critical field strengths vs. temperature in Fig. 2, a linear extrapolation down to temperature of $T=0 \mathrm{~K}$ as used by Skoskiewicz [17] would seem to be adequate. However, for reasons of the third law behavior, a parabolic extrapolation scheme was preferred:

$$
H_{\mathrm{c}}(T)=H_{\mathrm{c}}(0)\left[1-\left(\frac{T_{\mathrm{c}}(H)}{T_{\mathrm{c}}(0)}\right)^{2}\right] .
$$

Figure 4 summarizes the $H_{\mathrm{c}}(0)$ values, obtained by parabolic extrapolation, and, for the sake of comparison with Skoskiewicz's results on boron-free 


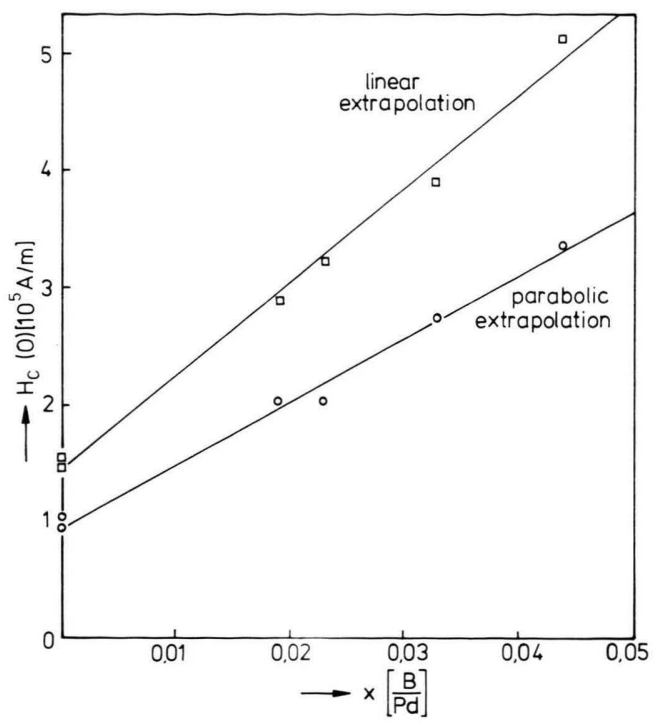

Fig. 4. Extrapolated critical field strengths of $\mathrm{PdB}_{x} \mathrm{H}_{y}$ alloys vs. boron content at $0 \mathrm{~K}$.

samples, also by linear extrapolation. The boronfree sample had a critical field strength at $0 \mathrm{~K}$ of $H_{\mathrm{c}}(0)=9.5510^{4} \mathrm{~A} / \mathrm{m}$. Horobiowski et al. [23] reported $8.7510^{4} \mathrm{~A} / \mathrm{m}$ - independent of hydrogen content. With increasing boron content $H_{\mathrm{c}}(0)$ increases linearly and reaches $3.310^{5} \mathrm{~A} / \mathrm{m}$ at $x=0.044$.

Palladium hydrogen samples, charged homogeneously by a high pressure method, are known to be type I superconductors [23]. The palladium-boronhydrogen samples of this work showed critical field strengths, which are too high for type I superconductivity. More detailed information was not ob-

[1] T. Skoskiewicz, Phys. Stat. Sol. (a) 11, K 123 (1972).

[2] B. Stritzker and W. Buckel, Z. Phys. 257,1 (1972).

[3] B. N. Ganguly, Z. Phys. B 22, 127 (1975).

[4] A. Eichler H. Wühl, and B. Stritzker, Solid state Comm. 17, 213 (1975).

[5] B. N. Ganguly, Phys. Rev. B 14, 3848 (1976).

[6] B. Stritzker and J. Becker, Phys. Lett. 51 A (3), 147 (1975).

[7] A. W. Szafranski, T. Skoskiewicz, and B. Baranowski, Phys. Stat. Sol. (a) 37, K 163 (1976).

[8] H. Husemann and H. Brodowsky, Z. Naturforsch. 23 a, 1693 (1968).

[9] H. Brodowsky, H. Husemann, and R. Mehlmann, Ber. Bunsenges. Phys. Chem. 77, 36 (1973).

[10] M. Mahnig and L. E. Toth, Phys. Lett. 32 a, 379 (1970).

[11] A. C. Switendick, Ber. Bunsenges. Phys. Chem. 76, 535 (1972)

[12] H. Brodowsky, H.-J. Schaller, and H.-J. Wernicke, Z. Metallkde. 70,631 (1979).

[13] H. Brodowsky and H.-J. Schaller, Ber. Bunsenges. Phys. Chem. 80, 656 (1976). tainable by the simple ac bridge. That value was taken to be the critical field strength, which raised the resistivity of a superconducting sample to half of the normal resistivity.

Extremely disordered $\mathrm{Pd}-\mathrm{H}$ alloys, as obtained by vapor deposition of palladium on solid hydrogen, were observed to be type II superconductors [24]. Thus it can be concluded, that scattering of conduction electrons by disordered palladium atoms or boron atoms, respectively, is effective enough to shorten the mean free path sufficiently to bring about type II superconductivity. A statistical distribution of hydrogen atoms and unoccupied octahedral sites, however, as in substoichiometric Pd-H produces a weaker electron scattering leading to type I superconductivity.

\section{Summary}

Palladium-boron-hydrogen alloys show superconductivity over a wide range of composition. The unique features of palladium alloys permit the preparation of samples with almost identical electronic structure but with varying $\mathrm{H}$ and $\mathrm{B}$ content. The isolated influence of atomic masses and elastic properties on the transition temperature has been investigated. No inverse mass effect as in Pd-H(D) alloys was observed. Measurements of critical field strengths gave evidence for type II superconductivity at higher boron concentrations.

\section{Acknowledgements}

We wish to thank the Deutsche Forschungsgemeinschaft for financial support.

[14] R. J. Soulen, J. F. Schooley, and G. A. Evans, Rev. Sci. Instr. 44, 1537 (1973).

[15] J. Fleischhauer, Dissertation Univ. Kiel 1981

[16] J. M. E. Harper, Phys. Lett. 47 A, 69 (1974).

[17] T. Skoskiewicz, Phys. Stat. Sol. (b) 59, 329 (1973).

[18] J. E. Schirber and C. J. M. Northrup, Jr., Phys. Rev. B 10, 3818 (1974).

[19] M. Zimmermann, G. Wolf, and K. Bohmhammel, Phys. Stat. Sol. (a) 31, 5121 (1975).

[20] D. A. Papaconstantopoulos, B. M. Klein, E. N. Economou, and L. L. Boyer, Phys. Rev. B 17, 141 (1978).

[21] P. C. Aben and W. G. Burgers, Trans. Faraday Soc. 58, 1989 (1962).

[22] H. Brodowsky and H.-J. Schaller, Trans. Met. Soc. Aime 245, 1015 (1969).

[23] M. Horobiowski, T. Skoskiewicz, and E. Trojnar, Phys. Stat. Sol. (b) 79, K 147 (1977).

[24] R. Mersevey and P. M. Tedrow, Bull. Amer. Phys. Soc. 21, 340 (1976). 